\title{
NÍVEL DE GOVERNANÇA CORPORATIVA PREDIZ O DESEMPENHO FINANCEIRO DA EMPRESA? EVIDÊNCIAS DO MERCADO BRASILEIRO POR MEIO DE REDES NEURAIS ARTIFICIAIS
}

\author{
DOES THE LEVEL OF CORPORATE GOVERNANCE PREDICTS THE \\ FINANCIAL PERFORMANCE OF THE COMPANY? EVIDENCE FROM THE \\ BRAZILIAN MARKET THROUGH ARTIFICIAL NEURAL NETWORKS
}

\author{
CLÁUDIA OLÍMPIA NEVES MAMEDE MAESTRI \\ Universidade Federal de Uberlândia. Endereço: Av. João Naves de Ávila, \\ 2121 | Saraiva | 38400-902 | Uberlândia/MG | Brasil. \\ (1) http://orcid.org/0000-0002-6978-5219 \\ claudiamamede@yahoo.com.br
}

\section{VITOR BORGES TAVARES}

Instituto Federal do Triângulo Mineiro. Endereço: Rua Blanche Galassi, 150 | Morada da Colina | 38411-104 | Uberlândia/MG | Brasil.

(1) http://orcid.org/0000-0002-7234-3761

vitorbtavares@gmail.com

\author{
ANTÔNIO SÉRGIO TORRES PENEDO \\ Universidade Federal de Uberlândia. Endereço: Av. João Naves de Ávila, \\ 2121 | Saraiva | 38400-902 | Uberlândia/MG | Brasil. \\ (1) http://orcid.org/0000-0001-7763-8457 \\ astpenedo@yahoo.com.br
}

\section{VINÍCIUS SILVA PEREIRA}

Universidade Federal de Uberlândia. Endereço: Av. João Naves de Ávila, 2121 | Saraiva | 38400-902 | Uberlândia/MG | Brasil.

(D) http://orcid.org/0000-0001-8439-7540

viniciuss56@gmail.com

\section{ROONEY RIBEIRO ALBUQUERQUE COELHO}

Universidade Federal de Uberlândia. Endereço: Av. João Naves de Ávila, 2121 | Saraiva | 38400-902 | Uberlândia/MG | Brasil.

D http://orcid.org/0000-0002-8792-6054

rooneycoelho@hotmail.com

\section{RESUMO}

Este estudo teve como objetivo desenvolver modelos de redes neurais artificiais para prever o desempenho financeiro das empresas pertencentes e não pertencentes aos níveis de governança da B3 (antiga BM\&FBovespa). Os modelos foram desenvolvidos utilizando dados das empresas do mercado de ações brasileiro no período de 2005 a 2017. Estipulou-se como hipótese que os modelos que corresponderiam às empresas pertencentes aos segmentos de governança obteriam maior sucesso na previsão do desempenho financeiro das empresas em comparação aos que 
representariam as que não estavam nos níveis de governança. Os resultados confirmaram a hipótese. Além disso, eles indicaram que o desempenho financeiro das empresas não pertencentes aos níveis de governança corporativa é mais sensível a oscilações ocorridas no ambiente externo, o que dificulta a previsão desse indicador. Este trabalho foi o primeiro no Brasil a construir modelos por meio de técnicas de inteligência artificial - mais especificamente a rede neural backpropagation - para prever o desempenho financeiro da empresa relacionando-o a aspectos da governança corporativa.

Palavras-chave: Desempenho Financeiro. Governança Corporativa. Redes Neurais Artificiais.

\begin{abstract}
This study aimed to develop models of artificial neural networks to predict the financial performance of companies in and outside the governance levels of the B3 (before, BM\&FBovespa). The models were developed using data from companies in the Brazilian stock market from 2005 to 2017. For these models, it was stipulated as a hypothesis that the models, which corresponded to the companies in the governance segments, would be most successful in predicting the performance of companies than those, which represent the ones that were not in the levels of governance. The results confirmed the hypothesis. In addition, they indicated that the performance of not belonging to corporate governance levels companies is more sensitive to oscillations in the external environment, making it difficult to forecast this indicator. This work was the first in Brazil to construct models through techniques of artificial intelligence - more specifically neural backpropagation network - to predict the performance of the company by relating it to aspects of corporate governance.
\end{abstract}

Keywords: Financial Performance. Corporate Governance. Artificial Neural Networks.

\title{
1 INTRODUÇÃO
}

Em um cenário de fraudes corporativas como o vivenciado pelos Estados Unidos com as empresas Enron, Global Crossing e Bernard L. Madoff, pelo Brasil com o Banco Santos, Boi Gordo, Daslu (Costa \& Wood Jr, 2012) e, mais recentemente, em meados da segunda década do século XXI, com as empresas OGX (Valor Econômico, 2015), Petrobras, Construtora Odebrecht (Estadão, 2015), entre outras, torna-se ainda mais relevante o estudo das práticas de governança corporativa.

As práticas de governança corporativa foram implantadas no Brasil em dezembro de 2000, com a inserção dos segmentos de governança corporativa e exigências que vão além das obrigações que as companhias têm perante a Lei das Sociedades por Ações (Lei n. 11.638/07). O objetivo dessa criação foi melhorar a avaliação das companhias que decidem aderir, voluntariamente, a um dos segmentos, e proporcionar um ambiente de negociação que estimule o interesse dos investidores e a valorização das companhias (BM\&FBovespa, 2019).

Neste contexto, Silveira, Leal, Carvalhal-da-Silva e Barros (2010) estudaram as pontuações gerais de governança corporativa de 1998 a 2004 avaliando os determinantes e a evolução de se aderir voluntariamente aos níveis de práticas de governança corporativa no Brasil. Os autores visualizaram que o nível de práticas de governança corporativa da empresa está em constante melhoria, mas ainda há muito espaço para avanços, que aderir voluntariamente a novos requisitos de listagem mais rigorosos está positivamente associado com melhorias no nível de práticas de governança corporativa da empresa e que reduzir ou não utilizar ações sem direito a voto melhora as práticas de governança corporativa.

Também surgiram diversos estudos sobre a relação entre a adoção de práticas de governança e o desempenho financeiro das empresas. Dentre eles, destacam-se os de Srour (2005), 
Okimura, Silveira e Rocha (2007), Macedo e Corrar (2012), Ferreira, Santos, Lopes, Fonseca e Nazareth (2013) e Catapan e Colauto (2014), tendo como suporte a utilização de técnicas estatísticas, como regressão logística, Monte Carlo, análise multivariada e a utilização de técnicas de programação matemática, como a análise por envoltória de dados (DEA).

Além desses estudos, destaca-se o trabalho de Chi (2009) com desenvolvimento de uma rede neural para analisar os índices de governança corporativa em Taiwan. As redes neurais artificiais (RNAs), de acordo com Zahedi e Rounaghi (2015), são modelos computacionais, inspirados no sistema nervoso central, utilizados para estimar ou aproximar funções que podem depender de um grande número de entradas, geralmente desconhecidas, sendo comumente apresentadas como sistemas de neurônios interconectados, que podem calcular valores de entradas, de uma maneira que os neurônios de entrada alimentam os valores para cada um dos neurônios na camada oculta, e a camada oculta fornece-os para a camada de saída.

O desempenho financeiro das empresas e algumas das variáveis que causam impacto nele estão sujeitos a oscilações, devido à ação da entropia dos sistemas. Nesses casos, segundo Burrell e Folarin (1997), as técnicas estatísticas tradicionais podem não ser eficazes para prever o comportamento de indicadores econômicos e financeiros.

A previsão é um fator crucial nos processos de tomada de decisão das empresas. Ela permite estimar resultados incontroláveis e concorre para a melhor escolha entre as alternativas possíveis. Em função de sua importância, as previsões financeiras e econômicas atraem o interesse de pesquisadores. Durante as últimas décadas, surgiram várias abordagens que diferem entre si no que diz respeito a metas de previsão, natureza das informações utilizadas e aparato matemático (Bodyanskiy \& Popov, 2006).

No que tange a técnicas de previsão, os modelos de redes neurais artificiais têm performance superior em comparação com outras técnicas (Oliveira, Nobre \& Zárate, 2013). Segundo Chen, Huang e Kuo (2009), esse método utiliza a experiência do passado para aprender sobre o problema e utiliza a lógica difusa para descrevê-lo, de forma similar ao cérebro humano, e tratar a imprecisão e incerteza associadas aos dados.

Nos últimos anos, as redes neurais artificiais desenvolveram modelos de pesquisa bemsucedidos para prever, detectar e resumir a estrutura das variáveis de mercados financeiros, sem confiar demais em pressupostos específicos e distribuições de erro (Duan \& Stanley, 2011).

Assim, o presente estudo teve como objetivo desenvolver modelos de redes neurais artificiais para prever o desempenho financeiro das empresas pertencentes e não pertencentes aos níveis de governança da B3 (ex-BM\&FBovespa), sendo este trabalho o primeiro no Brasil a construir modelos por meio de técnicas de inteligência artificial - mais especificamente rede neural backpropagation - para prever o desempenho financeiro da empresa em função de aspectos da governança corporativa e características específicas da empresa.

A abordagem de rede neural backpropagation é particularmente útil em estudos deste tipo, por fornecer uma revisão independente com aprovação do modelo de previsão e poder aumentar o âmbito de aplicação, a confiabilidade e a capacidade de previsão do modelo, além de facilitar a quantificação, comparação da influência e contribuição de dados de campo no processo de modelagem, o que ajuda a prever o desempenho financeiro da empresa baseado no mercado (Chi, 2009).

Além desta introdução, apresenta-se, na segunda seção, a fundamentação teórica que permitiu a compreensão literária sobre Governança Corporativa e sua relação com o desempenho financeiro das empresas. Na terceira, são evidenciados os métodos da pesquisa e, na quarta, discutidos os resultados. Por fim, realiza-se as considerações finais da pesquisa, na quinta seção.

\section{FUNDAMENTAÇÃO TEÓRICA}

A governança corporativa é um conjunto de mecanismos pelos quais os provedores de capital garantem para si a obtenção do retorno sobre seu investimento e os investidores externos 
se protegem contra a expropriação por parte dos insiders (Shleifer \& Vishny, 1997; La Porta, Lopez-de-Silanes, Shleifer \& Vishny, 2002). Segundo o Instituto Brasileiro de Governança Corporativa (IBGC, 2015), é um sistema pelo qual as organizações são dirigidas, monitoradas e incentivadas, envolvendo as práticas e os relacionamentos entre proprietários, conselho de administração, diretoria e órgãos de controle.

No Brasil, de acordo com o IBGC (2015), as boas práticas de governança corporativa permitem o alinhamento de interesses, a preservação e a otimização do valor da empresa o que facilita seu acesso ao capital, contribuindo, dessa forma, para a sua longevidade.

Estudos sobre as práticas de governança corporativa relacionados a variáveis como: volatilidade do retorno de ações, valor de mercado da empresa, custo de capital, desempenho financeiro e eficiência, têm contribuído para esclarecimento se a adesão aos segmentos de listagem de governança corporativa proporciona mais benefícios do que custos para as empresas.

Malacrida e Yamamoto (2006) analisaram se o nível de evidenciação de informações contábeis, apresentadas pelas empresas componentes do Ibovespa, influencia a volatilidade do retorno de suas ações quando negociadas na Bolsa de Valores de São Paulo, pois se espera que empresas com maior nível de evidenciação apresentem menor volatilidade dos retornos de suas ações. Foram coletadas informações publicadas por 42 empresas pertencentes ao Ibovespa, através dos relatórios anuais no período de 02.01.2002 a 30.04.2003. Após segregar essas companhias em 3 grupos distintos, de acordo com seus níveis específicos de evidenciação (maior, médio e menor), foram aplicados testes estatísticos com o propósito de se verificar a existência de diferenças significativas entre o nível de evidenciação das empresas e a volatilidade do retorno das suas ações. Verificou-se que maior nível médio de evidenciação resulta em menor volatilidade média dos retornos das ações.

Black, Carvalho e Sampaio (2014) reportaram as práticas de governança de empresas brasileiras em 2004, 2006, e 2009, para construir um amplo índice de governança corporativa e analisar a evolução da governança corporativa no Brasil e a associação entre governança e valor da empresa. Acharam que as práticas de governança corporativa melhoraram significativamente ao longo deste período. Esta evolução deve-se a dois fatores principais: 1) crescimento nas listagens Novo Mercado e Nível II (NM e L2), principalmente através de IPOs de novas empresas, e 2) melhores práticas em empresas não pertencentes ao NM e L2, principalmente através da adoção de elementos de governança necessários para a listagem NM e L2. As práticas de governança para as empresas já listadas em NM e L2 foram estáveis. A adoção dos elementos do índice de governança, desenvolvido pelos autores, é necessária para a listagem NM e L2 e prevê maior valor da empresa. Em contraste, a adoção dos restantes elementos do índice não prevê o valor da empresa. Assim, mudanças de governança parecem responder às preferências dos investidores.

Nesse contexto, Tavares e Penedo (2018) verificaram que a prática de governança das empresas é diretamente proporcional ao interesse em fazer adesão aos segmentos de governança corporativa da B3. Ou seja, as companhias que adotam mecanismos mais exigentes de governança aderem mais, proporcionalmente, aos níveis de governança corporativa da bolsa brasileira em comparação com as que praticam menos governança.

Catapan e Colauto (2014) tiveram como objetivo examinar se existe relação entre a governança corporativa e o desempenho econômico-financeiro em empresas brasileiras listadas na BM\&FBOVESPA (atual B3), considerando os anos de 2010-2012. Para tanto, os dados foram recolhidos junto ao DIVEXT - sistema de divulgação de informações da Comissão de Valores Mobiliários (CVM) e o software Empresas Net - também da CVM, com aplicação de regressão com dados em painéis para analisar as relações. O resultado evidenciou uma relação direta entre valor de mercado das empresas e nível de divulgação, ilustrando que quanto maior a evidenciação de informações, maior o valor de mercado das empresas. Observou-se também relação entre Índice de Governança Corporativa e duas variáveis: q de Tobin e Return On Assets-ROA. 
Almeida e Dalmácio (2015) investigaram como a concorrência no mercado do produto e o nível de governança corporativa afetam a precisão das previsões dos analistas. Usando uma amostra de empresas do mercado de ações brasileiro cobertas por analistas, descobriram que a concorrência nos mercados de produtos fornece incentivos para aumentar o fluxo de informação, mas não necessariamente para melhorar sua qualidade. No entanto, uma forte governança corporativa melhora o processo de informação financeira e, consequentemente, a qualidade das previsões dos analistas. A principal evidência do estudo mostra que os analistas que acompanham empresas em setores altamente competitivos e com forte governança corporativa são os mais precisos. Outra evidência do estudo é que a governança corporativa por si só contribui para melhorar a precisão dos analistas e reduzir o desvio de previsões, reforçando a ideia de que desempenha um papel relevante em ambientes institucionais fracos, como o contexto brasileiro. Assim, a qualidade do ambiente informacional é reforçada quando as empresas implementam mecanismos de governança corporativa que visam melhorar a qualidade do processo de informação financeira.

Badele e Fundeanu (2014) estudaram a Romênia, mercado em que a governança começou, em termos conceituais e regulamentos, no início dos anos 2000, pelo fato de que os aspectos políticos, jurídicos, econômicos e sociais foram desenvolvidos lentamente e com dificuldade. Os autores defendem que a boa governança corporativa agrega valor e ajuda a reduzir o custo de capital. Além disso, consideram que práticas de governança representam fatores importantes na atração de investimento e no aumento do desempenho financeiro e da competitividade a longo prazo.

O estudo de Huang, Cheng e Tseng (2014) teve como objetivo explorar o efeito dos mecanismos de governança (incluindo os controles formais e sociais) sobre o desempenho cooperativo comprador-fornecedor em cadeias de suprimento. A evidência empírica, obtida por meio de uma pesquisa realizada pelo correio com 106 empresas que participavam do "Centro de Sistema de Produção Satélite" taiwanês, indica que: (1) há uma relação em forma de U invertido entre um controle formal e o desempenho cooperativo; (2) controle social tem um efeito positivo sobre o desempenho cooperativo consistente; e (3) a utilização conjunta de controle formal e controle social poderia melhorar o desempenho cooperativo nas cadeias de abastecimento, mas apenas em casos com uso moderado de controle formal. Caso contrário, o controle social torna-se um fator de apoio que repara os danos do desempenho cooperativo de controle formal.

Nguyen, Locke e Reddy (2015) investigaram a relação entre a concentração de propriedade e o desempenho financeiro das empresas de Cingapura e Vietnã. Observaram como a relação é moderada pela qualidade da governança nacional ao considerar dois tipos diferentes de sistemas de governança nacionais (bem desenvolvido contra o subdesenvolvido). O efeito da concentração de propriedade no desempenho financeiro nesses mercados persiste mesmo após considerar a natureza dinâmica da relação. A descoberta apoia a previsão da teoria da agência sobre o efeito do controle eficaz dos grandes acionistas em mercados com propriedade altamente concentrada. Além disso, descobriram que a qualidade da governança nacional não importa quando explicada pela relação de concentração de propriedade no desempenho financeiro. O efeito positivo da concentração de propriedade sobre o desempenho financeiro das empresas que operam no sistema de governança nacional subdesenvolvido (Vietnã) tende a ser mais forte do que no sistema bem estabelecido (Singapura). Este resultado é consistente com o argumento de que a concentração da propriedade é um mecanismo de governança corporativa eficiente, que pode substituir a fraca qualidade da governança nacional.

Desta forma, tendo em vista que aspectos da governança corporativa propiciam menor volatilidade no retorno das ações (Malacrida \& Yamamoto, 2006), maior qualidade das previsões por parte dos analistas (Almeida \& Dalmácio, 2015) e melhor preservação e otimização do valor da empresa (IBGC, 2015), tem-se a hipótese deste trabalho: 
H1: os modelos com governança corporativa têm desempenho financeiro mais estável, apresentando menor erro no modelo de redes neurais.

\section{MÉTODO E DADOS}

O presente estudo teve como amostra as empresas brasileiras com ações listadas na B3 no período de 2005 a 2017, com exceção das instituições financeiras, por apresentarem estruturas contábeis e financeiras diferenciadas, como alto endividamento, baixo ativo imobilizado, alto capital circulante, dentre outras.

O ponto de partida deste estudo foi o trabalho de Chi (2009), que teve por objetivo construir um modelo de rede neural artificial conceitual para explicar a variação transversal no desempenho financeiro das empresas com Governança Corporativa em Taiwan. A partir desse, o presente estudo visou desenvolver um modelo de rede neural artificial para predizer o desempenho financeiro das empresas participantes e não participantes dos segmentos de listagem de governança corporativa no Brasil, cujas variáveis estão expostas na figura 1:

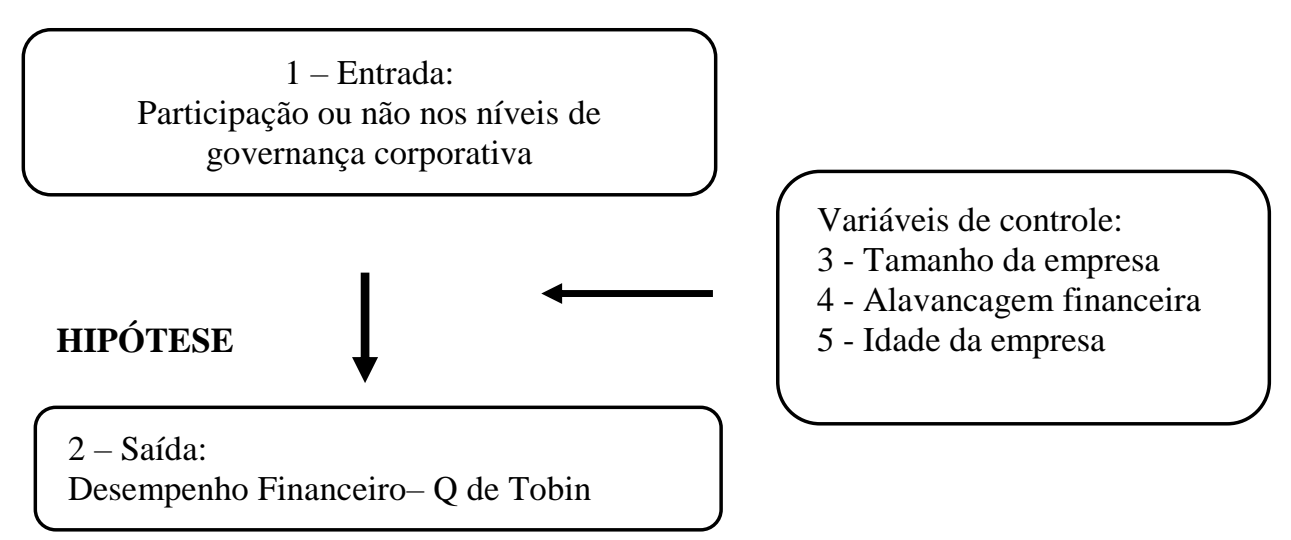

Figura 1. Modelo Teórico-Hipotético

Fonte: Elaborada pelos autores (2018)

Em relação às variáveis participantes do modelo, a variável independente foi segregada entre empresas participantes nos segmentos de listagem de governança com atribuição do valor um e empresas não participantes desses segmentos com atribuição do valor zero, sendo ambas informações coletadas da B3, conforme estudo de Maestri, Teruel e Ribeiro (2017) e Rodrigues e Ambrozini (2015).

A variável dependente formada pelo desempenho financeiro foi mensurada pelo método $\mathrm{Q}$ de Tobin, conforme estudos de Silva (2004), Leal (2004) e Catapan e Colauto (2014). Seu cálculo foi pela fórmula simplificada desenvolvida por Chung e Pruitt (1994) em que o desempenho financeiro da empresa é mensurado pela relação entre o valor de mercado da empresa e o valor de reposição de seus ativos, cujos dados foram coletados diretamente da base Economatica, conforme estudo de Ferreira et al. (2013).

As variáveis de controle foram o tamanho da empresa, a alavancagem financeira e a idade da empresa. O tamanho da empresa pode mensurar potenciais vantagens de escala, escopo e poder de mercado (Chi, 2009) e, conforme estudos de Silva (2004), Alencar (2007) e Chi (2009), foi calculado pelo logaritmo do ativo total. A alavancagem financeira pode mensurar o nível de endividamento da empresa (Silva, 2004), além disso, Watts \& Zimmerman (1990) e Tang (2010) consideram que uma empresa altamente alavancada está associada a um risco mais elevado e, portanto, seu desempenho financeiro fica enfraquecido. Os dados correspondentes a essas duas variáveis foram coletados diretamente na base de dados Economatica. A alavancagem financeira é calculada conforme Equação $1 \mathrm{ou}$, se o indicador do resultado financeiro for nulo, conforme Equação 2: 
$\mathrm{AF}=(\mathrm{LL}+\mathrm{PAM}) \times \mathrm{AT} /(\mathrm{PL}+\mathrm{PAM}) /(\mathrm{LL}+\mathrm{PAM}-\mathrm{RF})$

Equação (1)

Se RF é nulo, então:

$\mathrm{AF}=(\mathrm{LL}+\mathrm{PAM}) \times \mathrm{AT} /(\mathrm{PL}+\mathrm{PAM}) /(\mathrm{LL}+\mathrm{PAM}-\mathrm{RFA}-\mathrm{JPL}) \quad$ Equação $(2)$

Em que:

$\mathrm{AF}=$ alavancagem financeira;

$\mathrm{LL}=$ lucro líquido;

PAM = participação acionistas minoritários;

$\mathrm{AT}=$ ativo total;

$\mathrm{PL}=$ patrimônio líquido;

$\mathrm{RF}=$ resultado financeiro;

$\mathrm{RFA}=$ resultado financeiro do período anterior; $\mathrm{e}$

$\mathrm{JPL}=$ juros sobre o patrimônio líquido.

A idade da empresa, que está relacionada positivamente com boas práticas de governança (Ariff, Ibrahim \& Othman, 2007; Almeida \& Santos, 2008), foi obtida por meio de consulta individual no site do Cadastro Nacional de Pessoa Jurídica-CNPJ de cada empresa, sendo medida, conforme estudo de Chi (2009), como o logaritmo do número de anos desde a sua criação.

O método para análise desta pesquisa, assim como o de Chi (2009), foi o desenvolvimento de uma rede neural artificial. Esse método simula a forma como o cérebro humano realiza tarefas, por meio de componentes eletrônicos e programação em computadores. Assim como o cérebro, uma rede neural artificial é eficiente no armazenamento de conhecimento empírico e tem ótima capacidade de adaptação (Haykin, 2001).

As redes neurais possuem a capacidade de, a partir do aprendizado, generalizar a informação aprendida (Laboissiere, Fernandes \& Lage, 2015). Assim, ao propiciar uma generalização ótima de saída, as RNA minimizam a subjetividade no processo de tomada de decisão (Bodyanskiy \& Popov, 2006).

Em um estudo que averiguou a eficácia dos modelos de previsão de preço de ações em Istambul por meio de diferentes técnicas, Egeli, Ozturan e Badur (2003) verificaram que os modelos de RNA têm desempenho superior aos métodos clássicos de previsão de preços. Em consonância com esse resultado, Olson e Mossman (2003) obtiveram melhor precisão quando utilizaram redes neurais artificiais para prever o retorno das ações canadenses, em comparação com outras técnicas.

Leshno e Spector (1996) compararam a capacidade de previsão da rede neural com análise discriminante linear e análise discriminante quadrática, e os seus resultados indicam que a capacidade de previsão do modelo de rede neural é claramente mais precisa do que a dos modelos de análise discriminante clássicos.

Também fazendo um paralelo entre técnicas, Oliveira, Nobre e Zárate (2013) afirmam que, em comparação com as técnicas convencionais, as RNAs têm se mostrado mais eficientes em previsões de comportamento e tendências. Dessa forma, as redes neurais são técnicas eficazes para construções não-lineares e não-paramétricas, e superaram abordagens lineares, modelos econométricos e outros métodos convencionais em um grande número de problemas financeiros (Burrell \& Folarin, 1997).

No contexto da aprendizagem de uma rede neural, há vários algoritmos com essa finalidade, tendo como diferença entre eles a forma como modificam os pesos sinápticos de um neurônio. Em relação aos algoritmos, o mais utilizado nos trabalhos científicos tem sido o backpropagation (ou retropropagação), como nos trabalhos de Kolarik e Rudorfer (1994), Torres 
Jr, Machado e Souza (2005), Pao (2008), Chi (2009), Song, Liu e Chen (2012) e Tavares e Penedo (2018).

Segundo Haykin (2001), o algoritmo de retropropagação tem a função de ajustar os parâmetros livres da rede neural a fim de diminuir o erro da saída do modelo. Esse algoritmo inclui uma camada de entrada, uma ou mais camada(s) oculta(s) e uma camada de saída, representando um tipo de aprendizagem supervisionada, com aprendizagem por correção de erros que calcula um erro da camada de saída e propaga o erro para trás através da rede, para determinar a forma como cada um dos fatores de peso individuais contribui para o erro de saída (Haydar, Agdelen \& Özbeseker, 2006).

Quanto à estrutura do modelo de rede neural do presente trabalho, foi definida de acordo com Hecht-Nielsen (1990), o qual afirma que com apenas uma camada oculta já é possível calcular uma função qualquer a partir de dados fornecidos. A camada oculta realiza a intervenção entre a camada de entrada e a de saída de maneira útil, proporcionando extrações estatísticas de ordem elevada e com perspectiva global, decorrentes do acréscimo de conexões sinápticas e de interações neurais (Haykin, 2001). No modelo proposto por Hecht-Nielsen (1990), a camada oculta deve ter, aproximadamente, $2 \mathrm{i}+1$ neurônios, onde i é o número de variáveis de entrada. Assim sendo, o modelo desenvolvido no presente estudo compreendeu três variáveis de entrada (tamanho, alavancagem e idade), uma camada oculta composta por $7[(2 * 3)+1]$ neurônios e uma camada de saída (desempenho financeiro Q de Tobin) com um neurônio, conforme Figura 2.

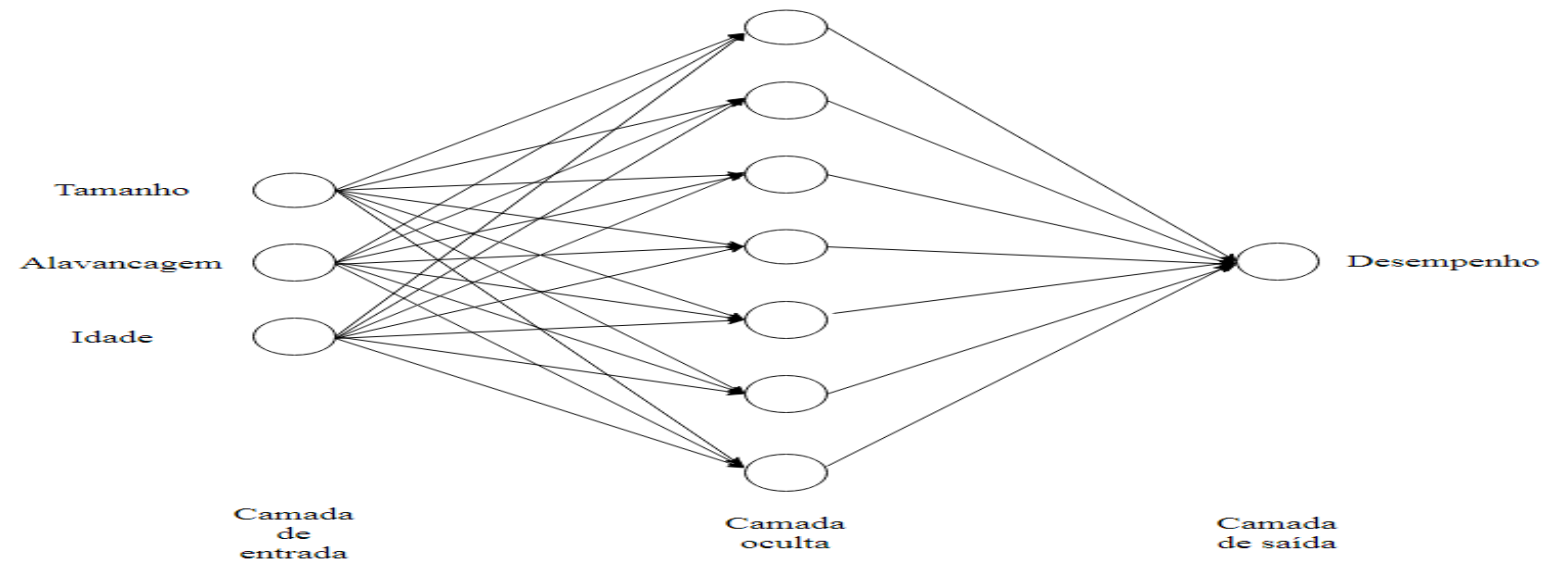

Figura 2. Modelo da rede neural

Fonte: Elaborada pelos autores (2018).

Para rodar o modelo, separou-se a amostra entre as empresas que tinham participação nos níveis de governança corporativa e as que não tinham.

\section{RESULTADOS}

As Tabelas 1 e 2 apresentam as estatísticas descritivas do presente estudo. A Tabela 1 contempla as variáveis de entrada e saída do modelo backpropagation da rede neural, com o resumo das observações, média, mediana, desvio-padrão, mínimo e máximo. Ressalta-se que a rede neural tem capacidade de se adaptar aos dados e por isso não houve necessidade de tratamento de outliers, por exemplo. 
Tabela 1

Estatística descritiva das variáveis do estudo (período: 2005 a 2017)

\begin{tabular}{llrrrrr}
\multicolumn{1}{c}{ Variáveis } & Obs & \multicolumn{1}{c}{ Média } & Mediana & Desvio Padrão & \multicolumn{1}{c}{ Mínimo } & \multicolumn{1}{c}{ Máximo } \\
\hline Governança & 2.865 & 0,4356 & 0,0000 & 0,4959 & 0,0000 & 1,0000 \\
Desempenho & 2.865 & 11,8387 & 0,5755 & 554,1977 & 0,0069 & $29.653,3100$ \\
Tamanho & 2.865 & 9,3813 & 9,3480 & 1,2037 & 3,2968 & 17,9144 \\
Alavancagem & 2.865 & 1,3599 & 1,5600 & 223,1222 & $-10.011,4100$ & $5.704,0200$ \\
Idade & 2.865 & 1,5050 & 1,6465 & 0,2880 & 0,5599 & 2,1004
\end{tabular}

Esta tabela fornece as variáveis participantes do modelo com o resumo das observações, média, desvio-padrão, mínimo e máximo, em que Governança foi definida em 1 para empresas participantes e 0 para as não participantes dos segmentos de listagem de Governança Corporativa; Desempenho mensurado pelo Q de Tobin pela relação entre o valor de mercado da empresa e o valor de reposição de seus ativos; Tamanho é medido pelo logaritmo do ativo total da empresa; Alavancagem Financeira é definida pela Equação 1 ou pela Equação 2; Idade é medida pelo logaritmo do número de anos desde o estabelecimento da empresa.

Fonte: Dados da pesquisa (2018).

Para a amostra estudada, evidencia-se na Tabela 1 o total de 2.865 observações, em que aproximadamente $44 \%$ das empresas da amostra participam de algum segmento (ou nível) de governança corporativa da B3. A média para o desempenho financeiro das empresas - mensurado pelo Q de Tobin - é de 11,8387 com mediana 0,5755, o que sugere que para a maioria de empresas da amostra, o mercado pode estar desvalorizando a empresa, pois seu valor de mercado é inferior ao seu valor de ativos. A média de tamanho das empresas, medida em logaritmo, é 9,3813 com mediana 9,3480, sinalizando valores de ativos de $\mathrm{R} \$ 2.406 .460 .301,00$ e $\mathrm{R} \$ 2.228 .445 .045,00$, respectivamente. A alavancagem financeira média é de 1,3599 com mediana 1,5600 que mostra que a maioria das empresas possui grau de alavancagem financeira maior que 1, ou seja, a maioria se endivida para alavancar seu negócio. A idade média das empresas, medida em logaritmo, é de 1,5050 com mediana 1,6465, o que evidencia que, entre o valor mínimo de 0,5599 (3 anos) e máximo de 2,1004 (123 anos), a idade da maioria das empresas é maior que 44 anos.

A Tabela 2 contempla a matriz de correlação das variáveis, permitindo a visualização de uma relação positiva entre tamanho e participação nos segmentos de governança corporativa e negativa, entre tamanho e idade e tamanho e desempenho financeiro. A variável alavancagem apresenta uma correlação negativa com idade. A variável idade apresentou uma relação negativa com participação nos segmentos de governança corporativa. Em suma, todas as variáveis com baixas correlações entre si indicam baixa endogeneidade e baixa autocorrelação.

\section{Tabela 2}

\section{Matriz de correlação das variáveis}

\begin{tabular}{|c|c|c|c|c|c|}
\hline Variáveis & Governança & Tamanho & Alavancagem & Idade & Desempenho \\
\hline Governança & 1,0000 & & & & \\
\hline Tamanho & $0,2487^{*}$ & 1,0000 & & & \\
\hline Alavancagem & $-0,0341$ & $-0,0080$ & 1,0000 & & \\
\hline Idade & $-0,0225^{*}$ & $-0,0302 *$ & $-0,0034^{*}$ & 1,0000 & \\
\hline Desempenho & $-0,0171$ & $-0,0785^{*}$ & $-0,0002$ & $-0,0318$ & 1,0000 \\
\hline
\end{tabular}

Esta tabela fornece a matriz de correlação das variáveis, em que Governança foi definida em 1 para empresas participantes e 0 para as não participantes dos segmentos de listagem de Governança Corporativa; Desempenho mensurado pelo $Q$ de Tobin pela relação entre o valor de mercado da empresa e o valor de reposição de seus ativos; Tamanho é medido pelo logaritmo do ativo total da empresa; Alavancagem Financeira é definida pela Equação 1 ou pela Equação 2; Idade é medida pelo logaritmo do número de anos desde o estabelecimento da empresa. (*) Significância de 0,05 .

Fonte: Dados da pesquisa (2018).

Estas variáveis participaram da rede neural backpropagation com $70 \%$ da amostra para treinamento, 15\% para validação e o restante de 15\% para teste. Segundo Song, Liu e Chen (2012), 
a amostra de treinamento envolve diretamente a formação da rede que continua fazendo a adoção e adaptação de acordo com o erro dessa amostra. A amostra de validação serve para testar a versatilidade da rede e parar a formação quando a versatilidade da rede não é mais optimizada. A amostra de teste testa o efeito de previsão da rede após a sua formação. A amostra total de previsão da rede neural backpropagation deste estudo foi de 2.865 dados, dos quais 2.005 foram para treinamento, 430 para verificação e 430 para teste.

Como resultado, os modelos desenvolvidos para prever o desempenho financeiro das empresas pertencentes e não pertencentes aos níveis de governança da B3 no período de 2005 a 2017 apresentaram, em média, erro quadrático médio (Mean Square Error-MSE) de 1,26 e 2,99, respectivamente. O melhor modelo de previsão anual para as empresas dos segmentos de governança teve como resultado um MSE de 0,24, e o pior modelo, 2,97; e para as empresas não participantes dos segmentos de governança o melhor MSE foi de 0,32 e o pior 8,72. A Figura 3 apresenta a comparação do erro de previsão dos modelos correspondentes às empresas participantes e não participantes dos segmentos de governança corporativa.

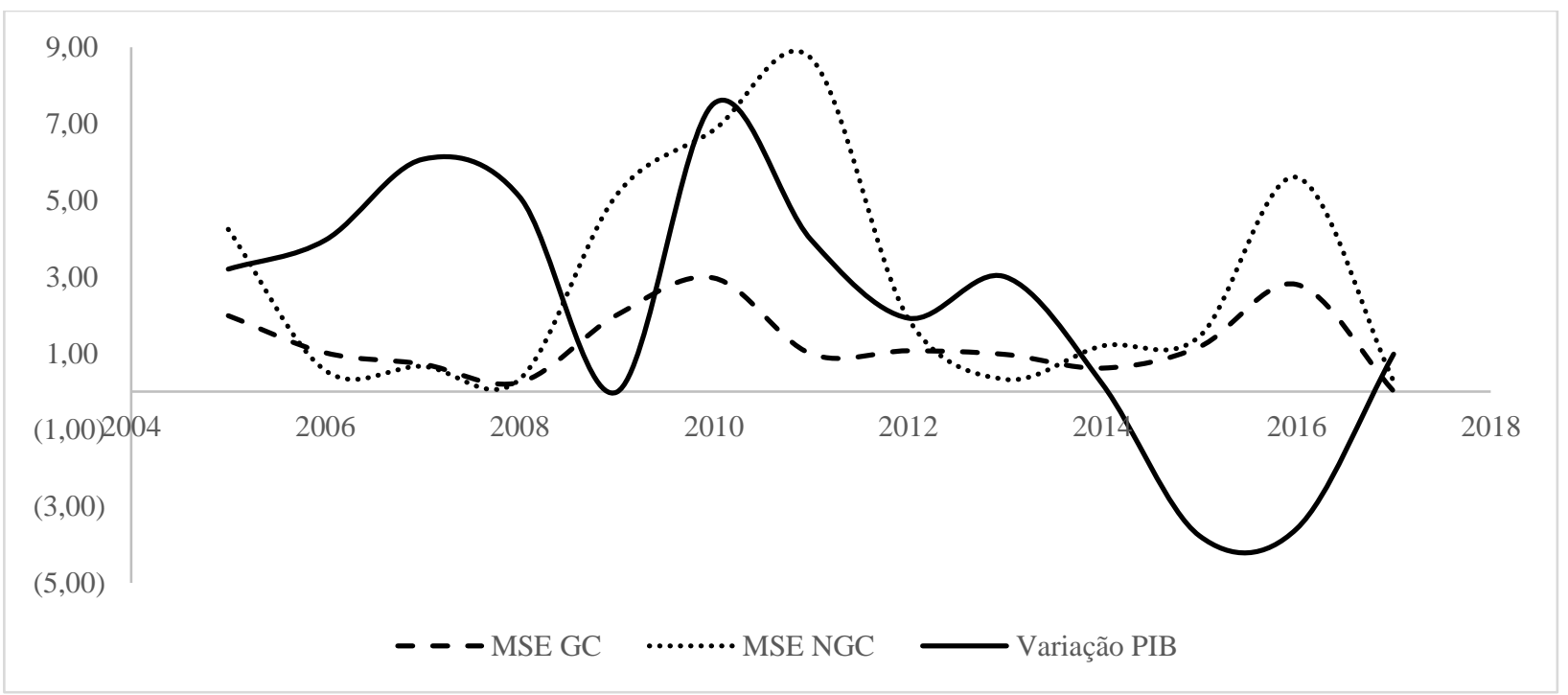

Figura 3. Resultados dos modelos de previsão, e comparação com a variação do PIB Fonte: Dados da pesquisa (2018).

Para retratar períodos de estabilidade e instabilidade econômicas, inseriu-se na comparação dos resultados entre os modelos a oscilação do PIB durante o período de estudo. Dessa forma é possível analisar como a previsão da rentabilidade das ações se dá em diferentes cenários macroeconômicos.

No período com estabilidade econômica (2005 a 2008 e 2011 a 2014), a previsão do desempenho financeiro das empresas listadas nos segmentos de governança corporativa foi similar, no que diz respeito à mensuração do erro quadrático médio, ao das não listadas, apresentando-se de forma ligeiramente mais estável em comparação aos modelos das empresas não pertencentes aos segmentos de governança corporativa. No entanto, no período de maior oscilação do PIB, a previsão do desempenho financeiro das empresas não participantes dos níveis de governança apresenta resultados significativamente mais instáveis em relação à dos modelos das empresas participantes.

Esse resultado indica que as empresas pertencentes aos níveis de governança corporativa têm maiores condições de enfrentar períodos de instabilidade e manter o desempenho financeiro perto do padrão. Diferentemente dessas, as empresas não pertencentes aos segmentos de governança são mais sensíveis a cenários com maiores mudanças no ambiente externo, o que faz com que seu desempenho financeiro oscile mais nessas circunstâncias, dificultando a previsão do desempenho financeiro. 
Assim, os resultados retratam a inferência feita acima de que a previsão do desempenho financeiro das empresas não listadas nos segmentos de governança corporativa é, significativamente, pior do que a das empresas listadas nesses segmentos em momentos de grandes oscilações no macroambiente.

Além disso, visualiza-se certa similaridade entre o sucesso da previsão que diz respeito à tendência de aumento ou queda do erro dos modelos. A diferença está no grau em que se dá o erro, significativamente maior em períodos instáveis para as empresas não participantes dos níveis de governança.

Ademais, os resultados sugerem que o tempo de percepção da (relativa) estabilidade no macroambiente é percebida pelas empresas pertencentes aos níveis de governança mais rápido. As empresas que não estão nesses segmentos têm um delay para compreender o cenário externo e aproximar o desempenho financeiro do padrão.

Ou seja, os fatores que dificultam a previsão causam impactos nos dois modelos (a correlação entre o erro dos modelos é alta, 0,63 , mas os modelos correspondentes às empresas não pertencentes aos segmentos de governança corporativa têm maior oscilação ao longo do tempo). Então, o desempenho financeiro das empresas pertencentes aos níveis de governança está sendo mais estável no decorrer do tempo.

\section{CONSIDERAÇÕES FINAIS}

Este trabalho desenvolveu modelos de redes neurais para realizar a previsão de desempenho financeiro das empresas pertencentes e não pertencentes aos níveis de governança corporativa. Para fazer isso, utilizou-se um método robusto e, comprovadamente, eficaz em estudos de previsão. Ao que se sabe, este foi o primeiro no Brasil que utilizou redes neurais para prever o desempenho financeiro de empresas no contexto de governança corporativa.

Os modelos desenvolvidos obtiveram bons resultados na previsão do desempenho financeiro das empresas, medido pelo Q de Tobin, com erro quadrático médio de 1,27, em média, para as empresas dos níveis de governança, e 2,86, para as empresas não listadas nos segmentos de governança corporativa.

Tem sido comum a realização de pesquisas que analisam como a governança corporativa altera a variação do desempenho financeiro, ou seja, se proporciona maior desempenho financeiro às empresas ou se elas têm resultados inferiores. Entretanto, este estudo analisou como a governança corporativa afeta a estabilidade do desempenho financeiro das empresas.

Nessa análise, verificou-se que os modelos de previsão de desempenho financeiro das empresas listadas nos segmentos de governança corporativa obtiveram, em média, melhor performance dos que os modelos correspondentes à previsão do desempenho financeiro das empresas não listadas nos segmentos de governança. Ademais, verificou-se que em períodos de crise o sucesso na previsão do desempenho financeiro das empresas não listadas nos segmentos de governança diminui significativamente em comparação aos modelos que contemplam as empresas listadas nos segmentos de governança corporativa.

Esses resultados sugerem que a governança corporativa contribui para a estabilidade do desempenho financeiro das empresas e para a capacidade de resiliência das organizações em cenários com alta intensidade de entropia. Ou seja, em cenários macroeconômicos instáveis, a boa governança corporativa permite à empresa estar melhor estruturada e manter mais estável o seu desempenho financeiro.

Em pesquisas futuras sugere-se analisar como se dá a variação no desempenho financeiro dos modelos de previsão nos diferentes níveis de governança corporativa da B3 (antiga BM\&FBovespa), pois acredita-se que as empresas do Novo Mercado possuam maior capacidade de manter estável seu desempenho financeiro ao longo dos anos, sofrendo menor pressão do ambiente externo. 


\section{REFERÊNCIAS}

Alencar, R. C. D. (2007). Nível de disclosure e custo de capital próprio no mercado Brasileiro. Tese de doutorado. Universidade de São Paulo, São Paulo, SP, Brasil.

Almeida, M. A., \& Santos, J. D. (2008). Relação entre variáveis endógenas e a qualidade das práticas de governança corporativa das empresas brasileiras de capital aberto não listadas em bolsa. Revista de Informação Contábil, 2(4), 17-37.

Almeida, J. E. F., \& Dalmácio, F. Z. (2015). The effects of corporate governance and product market competition on analysts' forecasts: evidence from the Brazilian capital market. The International Journal of Accounting, 50(3), 316-339.

Ariff, A. M., Ibrahim, M. K., \& Othman, R. (2007). Determinants of firm level governance: Malaysian evidence. Corporate Governance: The international journal of business in society, 7(5), 562-573.

Badele, C. S., \& Fundeanu, D. (2014). Policy's Beneficiaries of Corporate Governance and Diversification Strategy. Procedia-Social and Behavioral Sciences, 124, 468-477.

Black, B. S., Carvalho, A. G., \& Sampaio, J. O. (2014). The evolution of corporate governance in Brazil. Emerging Markets Review, 20, 176-195.

BM\&FBovespa (2019). Sobre segmentos de listagem. Segmentos de listagem. Recuperado em 27 maio, 2019, de http://www.bmfbovespa.com.br/pt_br/listagem/acoes/segmentos-delistagem/sobre-segmentos-de-listagem/

Bodyanskiy, Y., \& Popov, S. (2006). Neural network approach to forecasting of quasiperiodic financial time series. European Journal of Operational Research, 175(3), 1357-1366.

Burrell, P. R., \& Folarin, B. O. (1997). The impact of neural networks in finance. Neural Computing \& Applications, 6(4), 193-200.

Catapan, A., \& Colauto, R. D. (2014). Governança corporativa: uma análise de sua relação com o desempenho econômico-financeiro de empresas cotadas no Brasil nos anos de 2010-2012. Contaduría y Administración, 59(3), 137-164.

Chen, H. J., Huang, S. Y., \& Kuo, C. L. (2009). Using the artificial neural network to predict fraud litigation: Some empirical evidence from emerging markets. Expert Systems with Applications, 36(2), 1478-1484.

Chi, L. C. (2009). Do transparency and disclosure predict firm performance? Evidence from the Taiwan market. Expert Systems with Applications, 36(8), 11198-11203.

Chung, K. H., \& Pruitt, S. W. (1994). A simple approximation of Tobin's q. Financial management, $70-74$.

Costa, A. P. P. D., \& Wood Jr, T. (2012). Corporate frauds. Revista de Administração de Empresas, 52(4), 464-472. 
Duan, W. Q., \& Stanley, H. E. (2011). Cross-correlation and the predictability of financial return series. Physica A: Statistical Mechanics and its Applications, 390(2), 290-296.

Egeli B., Ozturan, M., \& Badur, B. (2003). "Stock market prediction using artificial neural networks". Proceeding of the Hawaii International Conference on Business, Honolulu, Hawaii, USA, 3.

Estadão (2015). Contrato da Petrobrás com a Odebrech é investigado por superfaturamento. $\begin{array}{lllll}\text { Recuperado em } & 29 & \text { junho, } & \text { de }\end{array}$ http://economia.estadao.com.br/noticias/negocios,contrato-da-petrobras-com-a-odebrechte-investigado-por-superfaturamento, $169716 \mathrm{e}$

Ferreira, R. N., Santos, A. C., Lopes, A. L. M., Fonseca, R. A., \& Nazareth, L. G. C. (2013). Governança corporativa, eficiência, produtividade e desempenho. Revista de Administração Mackenzie, 14(4).

Haydar, A., Agdelen, Z., \& Özbeseker, P. (2006). The use of backpropagation algorithm in the estimation of firm performance. Working paper, Istanbul Ticaret Üniversitesi.

Haykin, S. S. (2001). Redes neurais. Bookman.

Hecht-Nielsen, R. (1990). On the algebraic structure of feedforward network weight spaces. Advanced Neural Computers, 129-135.

Huang, M. C., Cheng, H. L., \& Tseng, C. Y. (2014). Reexamining the direct and interactive effects of governance mechanisms upon buyer-supplier cooperative performance. Industrial Marketing Management, 43(4), 704-716.

Instituto Brasileiro de Governança Corporativa - IBGC (2015). Recuperado em 29 junho, 2018, de http://www.ibgc.org.br/inter.php?id=18161/governanca-corporativa

Kolarik, T., \& Rudorfer, G. (1994). Time series forecasting using neural networks. ACM Sigapl Apl Quote Quad, 25(1), 86-94.

Laboissiere, L. A., Fernandes, R. A., \& Lage, G. G. (2015). Maximum and minimum stock price forecasting of Brazilian power distribution companies based on artificial neural networks. Applied Soft Computing, 35, 66-74.

La Porta, R., Lopez-de-Silanes, F., Shleifer, A., \& Vishny, R. W. (2002). Investor protection and corporate valuation. The Journal of Finance, 57(3), 1147-1170.

Leal, R. P. (2004). Governance practices and corporate value: a recent literature survey. Revista de Administração de Empresas da USP, 39(4), 327-337.

Lei $n$. 11.638/07. (2007). Altera e revoga dispositivos da Lei no 6.404, de 15 de dezembro de 1976, e da Lei no 6.385, de 7 de dezembro de 1976, e estende às sociedades de grande porte disposições relativas à elaboração e divulgação de demonstrações financeiras. Brasília, 2007. Recuperado em 28 abril, 2019, de http://www.planalto.gov.br/ccivil_03/_ato20072010/2007/lei/111638.htm 
Leshno, M., \& Spector, Y. (1996). Neural network prediction analysis: The bankruptcy case. Neurocomputing, 10(2), 125-147.

Malacrida, M. J. C., \& Yamamoto, M. M. (2006). Governança corporativa: nível de evidenciação das informações e sua relação com a volatilidade das ações do Ibovespa. Revista contabilidade e finanças, 17, 65-79.

Macedo, M. A. D. S., \& Corrar, L. J. (2012). Análise comparativa do desempenho contábilfinanceiro de empresas com boas práticas de governança corporativa no Brasil. Revista Contabilidade e Controladoria, 4(1).

Maestri, C. O. N. M., Teruel, R. L. H., \& Ribeiro, K. C. S. (2017). Governança Corporativa e o Impacto no Custo de Capital Próprio das Empresas Brasileiras de Capital Aberto. Revista de Finanças Aplicadas, 7(4), 1-17.

Nguyen, T., Locke, S., \& Reddy, K. (2015). Ownership concentration and corporate performance from a dynamic perspective: Does national governance quality matter? International Review of Financial Analysis, 41, 148-161.

Okimura, R. T., Silveira, A. D. M. D., \& Rocha, K. C. (2007). Estrutura de propriedade e desempenho corporativo no Brasil. RAC eletrônica, 1(1), 119-135.

Oliveira, F. A., Nobre, C. N., \& Zárate, L. E. (2013). Applying Artificial Neural Networks to prediction of stock price and improvement of the directional prediction index-Case study of PETR4, Petrobras, Brazil. Expert systems with applications, 40(18), 7596-7606.

Olson, D., \& Mossman, C. (2003). Neural network of Canadian stock returns using accounting ratios. International Journal of Forecasting, 19, 453-465.

Pao, H. T. (2008). A comparison of neural network and multiple regression analysis in modeling capital structure. Expert Systems with Applications, 35(3), 720-727.

Rodrigues, R. L., \& Ambrozini, M. A. (2015). Teoria de agência, política de dividendos e governança corporativa: evidências nas empresas brasileiras de capital aberto no período de 2000 a 2013. Anais do SEMEAD, São Paulo, SP, Brasil, 18.

Shleifer, A., \& Vishny, R. W. (1997). A survey of corporate governance. The journal of finance, 52(2), 737-783.

Silva, A. L. C. (2004). Governança corporativa, valor, alavancagem e política de dividendos das empresas brasileiras. Revista de Administração da Universidade de São Paulo, 39(4).

Silveira, A. D. M. D., Leal, R. P. C., Carvalhal-da-Silva, A. L., \& Barros, L. A. B. D. C. (2010). Endogeneity of Brazilian corporate governance quality determinants. Corporate Governance: International Journal of Business in Society, 10(2), 191-202.

Song, Z., Liu, D., \& Chen, S. (2012). A decision engineering method to identify the competitive effects of working capital: A neural network model. Systems Engineering Procedia, 5, 326333. 
Srour, G. (2005). Práticas diferenciadas de governança corporativa: um estudo sobre a conduta e a performance das firmas brasileiras. Revista Brasileira de Economia, 59(4), 635-674.

Tang, T. C. (2010). Effects of announcements of reorganization outcome. Applied Economics, 42(9), 1113-1124.

Tavares, V. B., \& Penedo, A. S. T. (2018). Níveis de governança corporativa da B3: interesse e desempenho das empresas - uma análise por meio de redes neurais artificiais. Revista Contabilidade, Gestão e Governança, 21(1), 40-62.

Torres Jr, R. G., Machado, M. A. S., \& Souza, R. C. (2005). Previsão de séries temporais de falhas em Manutenção industrial usando redes neurais. Engevista, 7(2), 4-18.

Valor Econômico. (2015). Polícia Federal investiga Eike Batista por três crimes contra o mercado. Recuperado em 29 junho, 2018, de http://www.valor.com.br/empresas/3520984/policiafederal-investiga-eike-batista-por-tres-crimes-contra-o-mercad

Zahedi, J., \& Rounaghi, M. M. (2015). Application of artificial neural network models and principal component analysis method in predicting stock prices on Tehran Stock Exchange. Physica A: Statistical Mechanics and its Applications, 438, 178-187.

Watts, R. L., \& Zimmerman, J. L. (1990). Positive accounting theory: A ten year perspective. The Accounting Review, 65(1), 131-156. 